\title{
Avaliação da toxicidade não-clínica do suco, da geleia e do iogurte da polpa de Pilosocereus gounellei
}

\author{
Evaluation of non-clinical toxicity of juice, jelly and yoghurt of Pilosocereus gounellei \\ Evaluación de la toxicidad no clínica del jugo, mermelada y yogur de pulpa Pilosocereus \\ gounellei
}

Wilma Ribeiro Coutinho de Almeida Guedes Pires ${ }^{1 *}$, Josué do Amaral Ramalho1, Igor Gabriel da Silva Ramalho ${ }^{1}$, Thyffanne Suellen Tavares Linhares ${ }^{1}$, Maria de Fátima Vanderlei de Souza ${ }^{1}$, Rita de Cássia Ramos do Egypto Queiroga ${ }^{1 * *}$, Caliandra Maria Bezerra Luna Lima ${ }^{1}$, Margareth de Fátima Formiga Melo Diniz ${ }^{1}$.

\section{RESUMO}

Objetivo: Realizar ensaios toxicológicos não-clínico agudo do suco, da geléia e do iogurte da polpa do Pilosocereus gounellei. Métodos: $O$ estudo foi realizado com 36 ratas Wistar, divididos em 3 grupos tratados e 3 controles, que receberam $2000 \mathrm{mg} / \mathrm{kg}$, via oral, dose única de cada substância. Foram observados por 14 dias, com relação ao consumo de água, ração e evolução ponderal. Ao fim do estudo, os animais foram eutanasiados e autopsiados. Foram avaliados parâmetros hematológicos, bioquímicos e realizado exames histopatológicos. Resultados: Não houve alteração no consumo de ração, porém os animais apresentaram diminuição de peso. Nos grupos do suco e geleia houve diminuição no consumo de água. No grupo do suco houve diminuição de ureia, aumento de cloro e magnésio. No grupo da geleia se evidenciou aumento de Alanina Aminotransferase (ALT) e Volume Corpuscular Médio. Naqueles que receberam o iogurte ocorreu diminuição de fósforo, magnésio, hematócrito e linfócitos. Todas estas alterações foram estatisticamente significativas, apesar de se encontrarem dentro dos padrões de normalidade para esta espécie, exceto com relação aos valores de ALT. Conclusão: Conclui-se que o suco e iogurte da geleia P.gounellei possuem baixa toxicidade. Em relação à geleia $P$. gounellei, mais estudos são necessários para descartar hepatotoxicidade.

Palavras-chave: Testes de toxicidade aguda, Cactaceae, logurte, Sucos.

\section{ABSTRACT}

Objective: Perform acute non-clinical toxicological tests of the juice, jelly and yogurt of the Pilosocereus gounellei. Methods: The study was carried out with 36 female Wistar rats, divided into 3 treated groups and 3 controls, who received $2000 \mathrm{mg} / \mathrm{kg}$, orally, of each substance. They were observed for 14 days, with respect to water consumption, food and weight evolution. Subsequently, the animals were euthanized and autopsied. Hematological and biochemical parameters were evaluated, anatomopathological examination was performed. Results: All animals showed weight loss, but there was no change in feed intake. In juice and jelly's groups, there was a decrease in water consumption. In the juice group there was a decrease in urea, lipase and an increase in chlorine and magnesium. In the jelly group, there was an increase in alanine aminotransferase and mean corpuscular volume. In those who received the yogurt there was a decrease in phosphorus and magnesium, hematocrit and lymphocytes. All of these changes were statistically significant, despite the values being within the normal range, except for ALT. Conclusion: It is concluded that the juice and yogurt of $P$. gounellei jelly are of low toxicity. Regarding $P$. gounellei jelly, further studies are needed to rule out hepatotoxicity.

Keywords: Toxicity tests acute, Cactaceae, Yogurt, Juices.

1 Universidade Federal da Paraíba (UFPB), João Pessoa - PB. *E-mail: wilminhaguedes@ hotmail.com
${ }^{* *}$ In memoriam 


\section{RESUMEN}

Objetivo: Realizar pruebas toxicológicas agudas no clínicas del zumo, mermelada y yogur de Pilosocereus gounellei. Métodos: El estudio se realizó con 36 ratas Wistar, divididas en 6 grupos (tratados y controles), que recibieron $2000 \mathrm{mg} / \mathrm{kg}$, vía oral, de cada sustancia. Se observo consumo de agua, alimentación y evolución del peso durante 14 días. Posteriormente, los animales fueron sacrificados y sometidos a autopsia. Se evaluaron parámetros hematológicos, bioquímicos y se realizó examen anatomopatológico. Resultados: Todos los animales mostraron pérdida de peso, pero sin cambios en la ingesta de alimento. En los grupos de zumo y mermelada, hubo una disminución en el consumo de agua. En el grupo de zumo hubo una disminución de urea, lipasa, aumento de cloro y magnesio. En el grupo de mermelada, hubo aumento en la alanina aminotransferasa y volumen corpuscular medio. En los que recibieron el yogur hubo una disminución de fósforo, magnesio, hematocrito y linfocitos. Todos estos cambios fueron estadísticamente significativos, pero los valores se encuentran dentro del rango normal, a excepción de ALT. Conclusión: Se concluye que el zumo y yogur de $P$. gounellei son de baja toxicidad. Con respecto a la mermelada de $P$. gounellei, se necesitan más estudios para descartar la hepatotoxicidad.

Palabras clave: Pruebas de toxicidad aguda, Cactaceae, Yogur, Zumos.

\section{INTRODUÇÃO}

A espécie Pilosocereus gounellei, pertencente à família Cactaceae, também conhecida como xique-xique, encontra-se dispersa pelo território brasileiro. Tem característica de forte adaptação às condições extremas de temperatura e umidade, multiplicando-se regularmente e cobrindo áreas extensas da caatinga (CARVALHO POAA, et al., 2021).

Estudos têm mostrado o potencial desta espécie, como fonte alimentar para comunidades do nordeste brasileiro (LUCENA CM, et al., 2012; SILVA VA, 2015). Na alimentação humana, o miolo dos cladódios é consumido cozido ou assado, sendo empregado também na produção de farinhas, biscoitos, bolos e doces. Na medicina popular, ele é consumido como suco, para tratamento de gastrite, colite e icterícia (LUCENA CM, et al., 2012).

Chaves EMF e Barros RFM (2015) citam que a utilização das cactáceas, na alimentação humana, restringe-se aos períodos de fome e seca, gerando efeitos negativos sobre sua utilização. Pesquisadores vêm estudando aspectos nutricionais do $P$. gounellei, sugerindo sua utilização, como fonte alimentar e de renda, orientando sua produção e aproveitamento em escala e estimulando o desenvolvimento de uma das regiões mais carentes do Brasil (PINTO NCC e SCIO E, 2014).

Estudos prévios com o suco do $P$. gounellei identificaram nutrientes importantes, como: fibras solúveis e insolúveis, e minerais (magnésio, cálcio, zinco, selênio e manganês), constituindo-o, uma alternativa alimentar de importante valor nutricional (BEZERRIL FF, et al., 2017). As fibras solúveis foram consideradas prebióticas (componentes alimentares não digeríveis que estimulam a proliferação e manutenção da microbiota intestinal) como mostrou um estudo que evidenciou efeito no crescimento de cepas do Lactobacillus (RIBEIRO TS, et al., 2020). Dezenove compostos fenólicos foram identificados no suco do P. gounellei, com destaque para os flavonoides e ácidos fenólicos (CARVALHO POAA, et al., 2021).

Resultados obtidos por Sousa GA, et al. (2018) mostraram, que o extrato etanólico do caule e da raiz do $P$. gounellei apresentaram atividade gastroprotetora em modelos de úlcera gástrica, induzidas por etanol em camundongos. Outro estudo com o suco do $P$. gounellei evidenciou efeito anti-inflamatório na mucosa intestinal de animais que foram submetidos à colite por ácido acético, com melhora do dano tecidual e diminuição de marcadores inflamatórios. Esses efeitos protetivos podem ser atribuídos aos compostos fenólicos e as fibras presentes no suco do xique-xique (CARVALHO POAA, et al., 2021).

No entanto, tem-se observado há alguns anos, que as plantas medicinais são consumidas com quase nenhuma comprovação de suas propriedades tóxicas. Sendo importante ressaltar que estes produtos também são passíveis de possuir efeitos adversos e toxicidade. Pesquisas mostraram que muitas plantas 
possuem substâncias potencialmente agressivas, devendo ser utilizadas com cuidado e respeitando seus riscos toxicológicos (MACIEL KS, et al., 2016; VERRI AM, et al., 2017).

A Toxicologia é uma ciência que estuda as propriedades físico-químicas das substâncias orientando a segurança de seu uso. Os estudos toxicológicos se concentram em duas fases: não clínica, realizada em espécies de experimentação e a clínica, onde os produtos que não demonstraram toxicidade significativa nos estudos não clínicos, são testados em humanos voluntários, sob rigorosos preceitos técnicos e éticos (DIAS GEN, 2015).

No Brasil, a toxicologia não clínica é orientada pelo Guia para a condução de estudos não clínicos de toxicologia e segurança farmacológica, necessários ao desenvolvimento de medicamentos, e pela Organização para Cooperação Econômica e Desenvolvimento (Organization for Economic Cooperation and Development-OECD) (SOUSA GA, et al., 2018).

Os ensaios toxicológicos não clínicos podem ser divididos em quatro fases: toxicidade aguda, de doses repetidas, subcrônica e crônica. A toxicidade aguda consiste em uma avaliação preliminar que objetiva conseguir dados acerca das características tóxicas da substância e seus efeitos adversos num organismo, permitindo inferir riscos para a saúde, resultantes de uma exposição de curta duração, sendo obrigatórios para toda substância em teste (OECD, 2001).

Diante disso, o objetivo deste estudo é realizar ensaios toxicológicos não clínico agudo do suco, da geleia e do iogurte de Pilosocereus gounellei. Durante a revisão da literatura, não foram encontrados estudos referentes à toxicidade destes produtos, justificando prosseguir com as análises toxicológicas não clínicas, sendo essa uma etapa fundamental para realização dos estudos clínicos.

\section{MÉTODOS}

Os procedimentos experimentais foram analisados e aprovados pelo Comitê de Ética em Pesquisa Animal (CEUA) da Universidade Federal da Paraíba (UFPB), protocolo $\mathrm{N}^{\circ} 1275210718$. Foram utilizadas 36 ratas Wistar (Ratus novergicus), albinas, adultas, nulíparas e não grávidas, pesando entre 180 e 220 gramas, fornecidas pela Unidade de Produção Animal (UPA) do Instituto de Pesquisa em Fármacos e Medicamentos (IPeFarM) UFPB. Estas, foram divididas em doze gaiolas de polietileno, contendo 3 animais em cada, e foram mantidas sob condições controladas de temperatura $\left(22 \pm 3^{\circ} \mathrm{C}\right)$, em ciclos de claro e escuro de 12 horas, com livre acesso à água e ração, e sem uso de medicamentos.

Foram utilizados seis grupos (três tratados e três controles) contendo seis animais cada um. Os testes foram realizados com três substâncias diferentes: suco do $P$. gounellei, cujo controle foi água; geleia do $P$. gounellei, sendo o controle uma geleia constituída de água e açúcar e iogurte acrescido da geleia do $P$. gounellei, cujo controle foi iogurte sem geleia. Depois de prontos, estes produtos, foram gentilmente cedidos pela professora doutora Rita Queiroga do Departamento de Nutrição da UFPB. O estudo foi desenvolvido, utilizando a dose de $2000 \mathrm{mg} / \mathrm{kg}$ de cada substância. A escolha da dose foi realizada, segundo recomendação da $O E C D$, seguindo os requisitos do teste limite.

O estudo de toxicidade aguda não clínica tem duração de 14 dias. No primeiro dia foi realizada a administração da substância em dose única, via oral, por gavagem, e a partir daí, observam-se os efeitos gerais apresentados pelos animais e diariamente se avalia o consumo de água e ração. Mamadeiras graduadas com água foram colocadas nas gaiolas pela manhã, sendo registrado no dia seguinte, o volume ingerido pelos animais. Quanto ao consumo de alimentos, a ração foi pesada e colocada nas gaiolas, sendo contabilizado, no dia seguinte, o peso consumido da mesma. A pesagem dos animais foi realizada semanalmente.

Antes da eutanásia e após jejum de 12 horas, amostras de sangue foram coletadas a partir do plexo braquial para análise de parâmetros hematológicos e bioquímicos. As análises hematológicas englobam contagem de hemácias, hematócrito, hemoglobina, volume corpuscular médio (VCM), hemoglobina corpuscular média $(\mathrm{HCM})$, concentração da hemoglobina corpuscular média $(\mathrm{CHCM})$ e amplitude de distribuição dos glóbulos vermelhos (RDW), contagem global dos leucócitos e diferenciação celular e 
plaquetas. As análises bioquímicas compreendem: glicose, ureia, colesterol total, triglicerídios, ácido úrico, creatinina, aspartato amino transferase (AST), alanina amino transferase (ALT), fosfatase alcalina (FA), amilase, desidrogenase lática (DHL), proteínas totais, sódio, potássio, cálcio, magnésio, fósforo e cloreto.

A eutanásia dos animais foi realizada ao fim dos 14 dias de observação, por sobredose de solução anestésica, contendo quetamina $(300 \mathrm{mg} / \mathrm{Kg})$ e xilazina $(30 \mathrm{mg} / \mathrm{Kg})$, segundo recomendação do Conselho Nacional de Controle de Experimentação Animal (CONCEA), e em seguida, realizou-se a autópsia. Foi efetuada a ressecção e pesagem dos pulmões, coração, estômago, fígado e rins. Os pulmões foram submetidos à perfusão, via traquéia, com solução de formol a 10\%, tamponada, enquanto os demais órgãos foram fixados em formalina tamponada. Após 72 horas, os órgãos foram resseccionados para processamento histopatológico.

A Pesquisa foi desenvolvida no Laboratório de Ensaios Toxicológicos (LABETOX) e no biotério Professor Thomas George do IPeFarM-UFPB. Para análise estatística dos resultados, foi utilizado o Teste "t" de Student não pareado, com o intuito de comparar as médias dos diferentes grupos em relação a cada parâmtero avaliado, utilizando o Software Graph Pad Prism 6.0, sendo os resultados considerados significativos quando apresentarem valores de $p<0,05$. Ressalta-se que os resultados foram expressos em média e erro padrão da média(e.p.m.).

\section{RESULTADOS}

Não foi observado nenhuma morte dos animais durante os 14 dias em que foi realizado o ensaio, apesar da alta dose administrada dos produtos, porém esta avaliação é apenas um indicativo de baixa toxicidade, sendo necessário estudos mais detalhados e de maior extensão, para avaliar uma possível toxicidade para a espécie, no seu uso continuado.

\section{Consumo de água e ração}

O consumo de água e ração foi avaliado diariamente, pela manhã, durante os 14 dias de experimento. $O$ grupo da geléia do P. gounellei apresentou um consumo de água significativamente menor que o grupo controle (geléia: $193,4 \pm 6,07$ e controle: $212,3 \pm 5,21$ ) como pode ser visto na tabela 1 . Os animais que receberam o suco também apresentaram diminuição do consumo de água comparado ao grupo controle (suco: 157,5 $\pm 4,96$ e controle: $175,5 \pm 6,38$ ). No grupo do iogurte não houve alteração em relação ao consumo de água e nem de ração. Não foi encontrada diferença estatística significativa no consumo de ração entre os grupos avaliados (Tabela 1).

Tabela 1 - Consumo de água e ração de Ratos Wistar, fêmeas, tratadas com a dose de $2000 \mathrm{mg} / \mathrm{kg}$ com o suco, geleia, iogurte com geleia do $P$. gounellei durante os 14 dias do ensaio de toxicidade aguda.

\begin{tabular}{ccccccc}
\hline \multicolumn{7}{c}{ GRUPO } \\
\hline CT-I & logurte & CT-G & Geleia & CT-S & Suco \\
\hline $\begin{array}{c}\text { ÁNSUMO DE } \\
\text { ÁGL) }\end{array}$ & $185,9 \pm 6,11$ & $\begin{array}{c}196,6 \pm 5,0 \\
0\end{array}$ & $212,3 \pm 5,21$ & $193,4 \pm 6,07^{*}$ & $175,5 \pm 6,38$ & $157,5 \pm 4,96^{*}$ \\
\hline $\begin{array}{c}\text { CONSUMO DE } \\
\text { RAÇÃO (g) }\end{array}$ & $111,8 \pm 5,02$ & $\begin{array}{c}105,4 \pm 3,8 \\
6\end{array}$ & $112,2 \pm 3,32$ & $105,9 \pm 2,41$ & $102,8 \pm 3,15$ & $113,8 \pm 3,88$ \\
\hline
\end{tabular}

Legenda: Os valores estão expressos em média \pm erro padrão da méida (e.p.m) ( $n=6)$. Teste " $t$ " de Student / Não-pareado. ${ }^{*} \mathrm{p}<0,05$. CT-I: controle do iogurte. CT-G: controle da geleia. CT-S: controle do suco.

Fonte: Pires WRCAG, et al., 2021.

\section{Evolução ponderal}

Com relação a evolução ponderal pode-se observar que durante os 14 dias de avaliação, os animais que receberam o iogurte da geleia apresentaram um ganho de peso menor comparado ao grupo controle, como pode ser visto no Gráfico $1 \mathrm{~A}$. Este grupo apresentou um delta de ganho de peso de $16,17 \mathrm{~g} \pm 1,78 \mathrm{~g}$, enquanto 
que o seu controle teve um ganho de $23 \mathrm{~g} \pm 1$,93g.Já o grupo da geléia apresentou uma variação de peso maior $(25,17 \mathrm{~g} \pm 4,28 \mathrm{~g})$, durante os 14 dias de experimento, comparado ao seu controle $(15,33 \mathrm{~g} \pm 1,65 \mathrm{~g})$, como pode ser visto no Gráfico 1 B. Ao avaliar o grupo do suco e seu controle, pode-se perceber que a variação de peso entre os dois foi semelhante, como mostra o Gráfico $1 \mathrm{C}$.

Gráfico 1 - Ganho de peso de Ratos Wistar, fêmeas, tratadas com a dose de $2000 \mathrm{mg} / \mathrm{kg}$, do suco, da geléia e do iogurte com geleia do $P$. gounellei, durante os 14 dias do ensaio de toxicidade aguda.
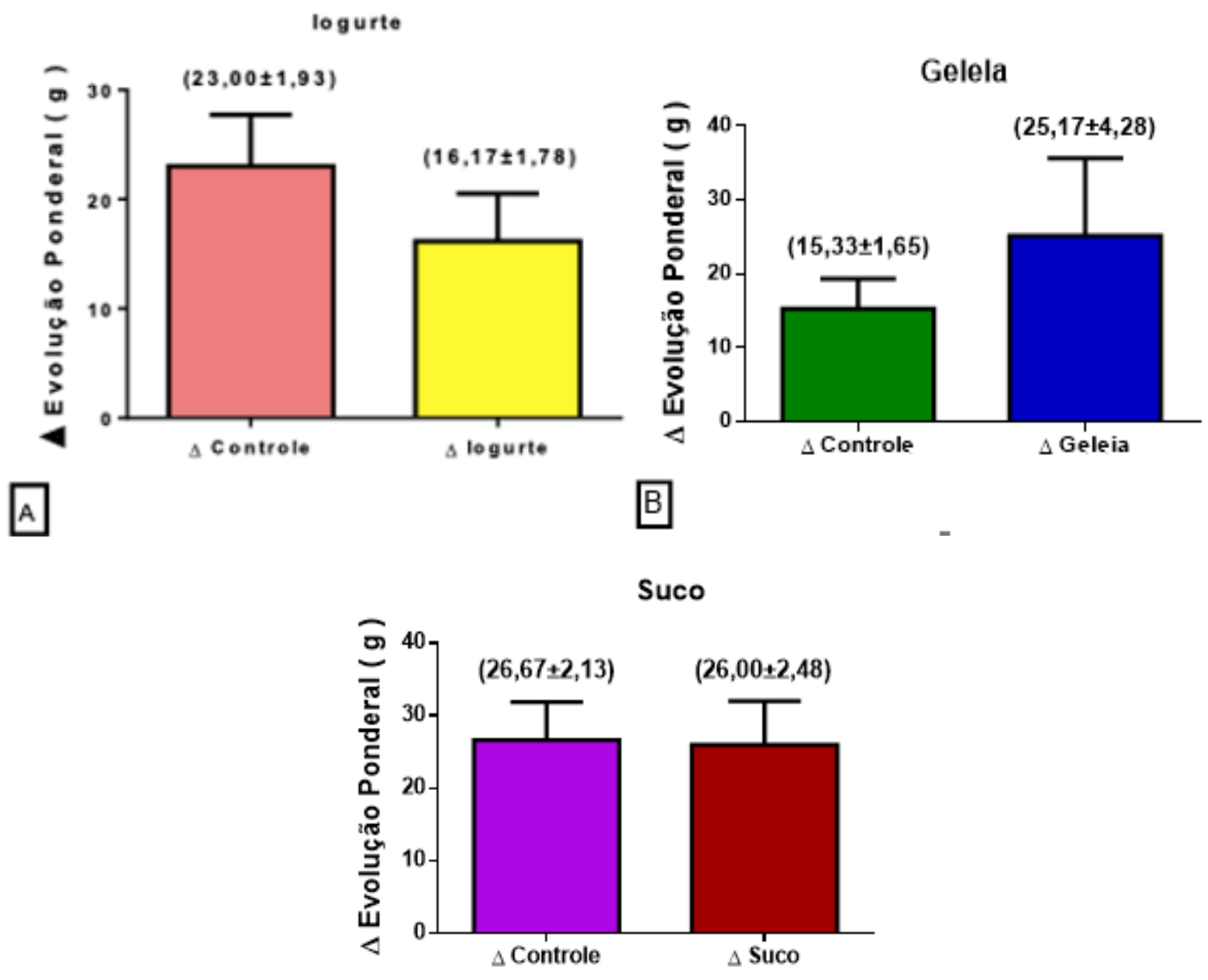

C

Legenda: A: Delta de ganho de peso de Ratos Wistar, fêmeas, tratadas com a dose de $2000 \mathrm{mg} / \mathrm{kg}$, de iogurte com geleia do $P$. gounellei, durante os 14 dias do ensaio de toxicidade aguda. B: Delta de ganho de peso de Ratos Wistar, fêmeas, tratadas com a dose de $2000 \mathrm{mg} / \mathrm{kg}$, com a geleia do $P$. gounellei, durante os 14 dias do ensaio de toxicidade aguda. C: Delta de ganho de peso de Ratos Wistar, fêmeas, tratadas com a dose de $2000 \mathrm{mg} / \mathrm{kg}$, com o suco do $P$. gounellei, durante os 14 dias do ensaio de toxicidade aguda.

Os valores estão expressos em média \pm erro padrão da média (e.p.m.) ( $n=6)$. Teste "t" de Student / Nãopareado. ${ }^{*} p<0,05$. Fonte: Pires WRCAG, et al., 2021.

\section{Parâmetros bioquímicos}

Poucas alterações foram evidenciadas na avaliação dos parâmetros bioquímicos, porém, algumas foram estatisticamente significativas, como pode-se verificar na Tabela 2. Dentre elas, observa-se um aumento de ALT no grupo que recebeu a geleia do $P$. gounellei. Houve diminuição nos níveis de ureia e lipase nos animais que receberam o suco do $P$. gounellei, além de um aumento significativo do cloro e do magnésio. No grupo que recebeu o iogurte com a geleia do $P$. gounellei, foi identificada uma diminuição significativa nos níveis de magnésio, fósforo e LDH em relação ao controle. 
Tabela 2 - Parâmetros Bioquímicos de Ratos Wistar tratados com a dose de $2000 \mathrm{mg} / \mathrm{kg}$ do suco, da geleia e do iogurte com a geléia do $P$. gounellei, durante o ensaio de toxicidade aguda.

\begin{tabular}{|c|c|c|c|c|c|c|}
\hline Grupos & CT- I & logurte & CT-G & Geleia & CT-S & Suco \\
\hline $\begin{array}{l}\text { Glicose } \\
\text { (mg/dL) }\end{array}$ & $124,7 \pm 2,2$ & $128,8 \pm 7,7$ & $149,3 \pm 1,1$ & $154,8 \pm 11,4$ & $104,0 \pm 6,7$ & $129,0 \pm 6,8$ \\
\hline Ureia $(\mathrm{mg} / \mathrm{dL})$ & $28,3 \pm 1,2$ & $32,5 \pm 2,5$ & $24,3 \pm 0,2$ & $25,8 \pm 0,7$ & $36,7 \pm 1,1$ & $31,5 \pm 1,5^{*}$ \\
\hline $\begin{array}{c}\text { Creatinina } \\
(\mathrm{mg} / \mathrm{dL})\end{array}$ & $0,44 \pm 0,03$ & $0,41 \pm 0,03$ & $0,38 \pm 0,01$ & $0,39 \pm 0,02$ & $0,37 \pm 0,02$ & $0,37 \pm 0,01$ \\
\hline $\begin{array}{l}\text { Colesterol } \\
(\mathrm{mg} / \mathrm{dL})\end{array}$ & $58,3 \pm 3,27$ & $53,7 \pm 3,69$ & $54,0 \pm 0,97$ & $55,8 \pm 6,18$ & $64,7 \pm 4,36$ & $59,8 \pm 4,06$ \\
\hline $\begin{array}{l}\text { Triglicerídeos } \\
\text { (mg/dL) }\end{array}$ & $33,3 \pm 3,29$ & $35,7 \pm 2,89$ & $32,3 \pm 2,43$ & $35,3 \pm 4,77$ & $26,0 \pm 0,97$ & $29,3 \pm 1,99$ \\
\hline $\begin{array}{l}\text { Ácido úrico } \\
\text { (mg/dL) }\end{array}$ & $1,1 \pm 0,19$ & $0,8 \pm 0,10$ & $1,5 \pm 0,46$ & $1,9 \pm 0,82$ & $0,7 \pm 0,06$ & $1,1 \pm 0,22$ \\
\hline $\begin{array}{l}\text { AST } \\
(\mathrm{U} / \mathrm{I})\end{array}$ & $193,3 \pm 46,0$ & $129,5 \pm 19,5$ & $79,33 \pm 2,6$ & $31,0 \pm 1,59$ & $119,3 \pm 2,1$ & $207,7 \pm 67,4$ \\
\hline $\begin{array}{l}\text { ALT } \\
\text { (U/I) }\end{array}$ & $37,67 \pm 2,76$ & $39,2 \pm 2,90$ & $239,3 \pm 101,1$ & $66,0 \pm 18,22^{*}$ & $47,0 \pm 4,21$ & $39,5 \pm 3,93$ \\
\hline $\begin{array}{c}\text { Fosfatase } \\
\text { Alcalina (U/I) }\end{array}$ & $123 \pm 7,73$ & $112,5 \pm 14,97$ & $100,7 \pm 10,42$ & $90,5 \pm 9,23$ & $143 \pm 19,75$ & $115,5 \pm 14,16$ \\
\hline $\begin{array}{l}\text { LDH } \\
(\mathrm{U} / \mathrm{I})\end{array}$ & $1656 \pm 36,27$ & $1301 \pm 88,28^{*}$ & $814 \pm 86,67$ & $1429 \pm 373,5$ & $1336 \pm 72,13$ & $1153 \pm 196,1$ \\
\hline $\begin{array}{l}\text { Amilase } \\
(\mathrm{U} / \mathrm{dL})\end{array}$ & $330,7 \pm 17,41$ & $312,8 \pm 15,57$ & $361,7 \pm 3,66$ & $339,3 \pm 13,56$ & $263,3 \pm 15,76$ & $297,0 \pm 16,66$ \\
\hline $\begin{array}{c}\text { Proteínas } \\
\text { totais }(\mathrm{g} / \mathrm{dL})\end{array}$ & $6,2 \pm 0,11$ & $5,88 \pm 0,12$ & $6,03 \pm 0,08$ & $6,12 \pm 0,09$ & $6,07 \pm 0,076$ & $5,87 \pm 0,140$ \\
\hline $\begin{array}{l}\text { Albumina } \\
(\mathrm{g} / \mathrm{dL})\end{array}$ & $3,13 \pm 0,06$ & $3,15 \pm 0,08$ & $3,06 \pm 0,06$ & $3,22 \pm 0,06$ & $3,23 \pm 0,056$ & $3,20 \pm 0,068$ \\
\hline $\begin{array}{l}\text { Globulina } \\
\text { (g/dL) }\end{array}$ & $3,07 \pm 0,14$ & $2,73 \pm 0,67$ & $2,73 \pm 0,67$ & $2,73 \pm 0,14$ & $2,83 \pm 0,095$ & $2,67 \pm 0,088$ \\
\hline $\begin{array}{l}\text { Bilirrubina } \\
\text { (mg/dL) }\end{array}$ & $0,13 \pm 0,02$ & $0,13 \pm 0,02$ & $0,16 \pm 0,02$ & $0,13 \pm 0,02$ & $0,17 \pm 0,021$ & $0,15 \pm 0,022$ \\
\hline $\begin{array}{l}\text { Cálcio } \\
\text { (mg/dL) }\end{array}$ & $9,97 \pm 0,12$ & $9,90 \pm 0,05$ & $9,97 \pm 0,10$ & $10,02 \pm 0,09$ & $10,07 \pm 0,042$ & $10,07 \pm 0,088$ \\
\hline $\begin{array}{l}\text { Cloro } \\
(\mathrm{U} / \mathrm{I})\end{array}$ & $101,7 \pm 0,21$ & $102,3 \pm 0,56$ & $101,7 \pm 0,21$ & $102,3 \pm 0,80$ & $100,7 \pm 0,21$ & $102,7 \pm 0,67^{*}$ \\
\hline $\begin{array}{l}\text { CPK } \\
(\mathrm{U} / \mathrm{I})\end{array}$ & $1727 \pm 538$ & $845,2 \pm 161,4$ & $440 \pm 18,62$ & $687,3 \pm 201,9$ & $672,2 \pm 28,86$ & $1001 \pm 230,8$ \\
\hline $\begin{array}{l}\text { Fósforo } \\
\text { (U/dL) }\end{array}$ & $6,37 \pm 0,24$ & $5,67 \pm 0,10^{*}$ & $6,23 \pm 0,27$ & $6,23 \pm 0,61$ & $6,53 \pm 0,055$ & $6,60 \pm 0,410$ \\
\hline $\begin{array}{l}\text { Lipase } \\
\text { (g/dL) }\end{array}$ & $5,0 \pm 0,63$ & $5,5 \pm 1,36$ & $5,67 \pm 1,05$ & $5,60 \pm 1,08$ & $6,33 \pm 0,76$ & $3.83 \pm 0,69^{*}$ \\
\hline $\begin{array}{l}\text { Magnésio } \\
\text { (g/dL) }\end{array}$ & $2,23 \pm 0,02$ & $2,03 \pm 0,07^{\star}$ & $2,20 \pm 0,07$ & $2,25 \pm 0,18$ & $1,93 \pm 0,02$ & $2,12 \pm 0,05^{\star}$ \\
\hline $\begin{array}{c}\text { Potássio } \\
\text { (g/dL) }\end{array}$ & $4,4 \pm 0,09$ & $4,23 \pm 0,11$ & $4,50 \pm 0,49$ & $4,55 \pm 0,40$ & $4,30 \pm 0,04$ & $4,53 \pm 0,31$ \\
\hline $\begin{array}{l}\text { Sódio } \\
\text { (g/dL) }\end{array}$ & $142 \pm 0,36$ & $142,3 \pm 0,33$ & $142,7 \pm 0,21$ & $141,7 \pm 0,49$ & $142,0 \pm 0,00$ & $142,3 \pm 0,33$ \\
\hline
\end{tabular}

Legenda: Os valores estão expressos em média \pm e.p.m. $(n=6)$. Teste " $t$ " de Student / Não-pareado. ${ }^{*}<0,05$. CT-I: controle do iogurte. CT-G: controle da geleia. CT-S: controle do suco. AST: aspartato aminotransferase. ALT: alanina aminotransferase. LDH: desidrogenase láctica. CPK: creatinofosfoquinase.

Fonte: Pires WRCAG, et al., 2021. 


\section{Parâmetros hematológicos}

Não foi observada diferença significativa entre os parâmetros hematológicos dos animais que receberam o suco do $P$. gounellei. No grupo tratado com a geleia do $P$. gounellei ocorreu um aumento estatisticamente significativo no VCM e no RDW em relação ao controle. Nas fêmeas que receberam o iogurte acrescido da geleia do $P$. gounellei houve uma diminuição do hematócrito, dos linfócitos e um aumento no RDW estatisticamente significativo em relação ao grupo controle (Tabela 3).

Tabela 3 - Parâmetros hematológicos de Ratos Wistar tratados com suco, geleia e iogurte com a geleia do $P$. gounellei na dose de $2000 \mathrm{mg} / \mathrm{kg}$ durante o ensaio de toxicidade aguda.

\begin{tabular}{|c|c|c|c|c|c|c|}
\hline Grupos & CT- I & logurte & CT- G & Geleia & CT-S & Suco \\
\hline $\begin{array}{l}\text { Hemácias } \\
\left(10^{6} \mathrm{~mm}^{3}\right)\end{array}$ & $\begin{array}{c}8,13 \pm 0,04 \\
4\end{array}$ & $8,05 \pm 0,180$ & $8,01 \pm 0,20$ & $8,05 \pm 0,23$ & $7,96 \pm 0,07$ & $7,64 \pm 0,12$ \\
\hline $\begin{array}{l}\text { Hemoglobina } \\
\text { (g/dl) }\end{array}$ & $14,2 \pm 0,15$ & $13,42 \pm 0,26$ & $13,9 \pm 0,16$ & $13,62 \pm 0,39$ & $13,90 \pm 0,12$ & $13,40 \pm 0,20$ \\
\hline $\begin{array}{c}\text { Hematócrito } \\
(\%)\end{array}$ & $41,1 \pm 0,35$ & $38,6 \pm 0,80^{*}$ & $40,44 \pm 0,50$ & $39,90 \pm 0,87$ & $39,76 \pm 0,36$ & $13,40 \pm 0,20$ \\
\hline $\begin{array}{l}\text { VCM } \\
\left(\mu^{3}\right)\end{array}$ & $\begin{array}{c}50,84 \pm 0,2 \\
8 \\
\end{array}$ & $48,08 \pm 1,67$ & $39,90 \pm 0,87$ & $49,52 \pm 0,34^{*}$ & $49,96 \pm 0,42$ & $50,94 \pm 0,63$ \\
\hline $\begin{array}{l}\mathrm{HCM} \\
(\mu \mu \mathrm{g})\end{array}$ & $\begin{array}{c}17,54 \pm 0,0 \\
92\end{array}$ & $39,90 \pm 0,87$ & $16,82 \pm 0,16$ & $16,82 \pm 0,16$ & $17,50 \pm 0,20$ & $17,52 \pm 0,20$ \\
\hline $\begin{array}{c}\mathrm{CHCM} \\
(\%)\end{array}$ & $\begin{array}{c}34,56 \pm 0,1 \\
0\end{array}$ & $39,76 \pm 0,36$ & $34,26 \pm 0,21$ & $34,26 \pm 0,21$ & $34,96 \pm 0,70$ & $34,42 \pm 0,24$ \\
\hline $\begin{array}{l}\text { Leucócitos } \\
(10 / \mathrm{mm})\end{array}$ & $\begin{array}{c}4160 \pm 712 \\
, 5 \\
\end{array}$ & $4100 \pm 148,3$ & $3780 \pm 563,4$ & $4300 \pm 876,4$ & $5240 \pm 815,2$ & $3360 \pm 510,5$ \\
\hline $\begin{array}{c}\text { Neutrófilo } \\
(\%)\end{array}$ & $15,2 \pm 1,71$ & $20,8 \pm 1,02$ & $16,6 \pm 0,93$ & $26,2 \pm 5,49$ & $20,0 \pm 3,35$ & $15,6 \pm 0,40$ \\
\hline $\begin{array}{c}\text { Eosinófilo } \\
(\%)\end{array}$ & $0,0 \pm 0,0$ & $0,2 \pm 0,2$ & $0,2 \pm 0,2$ & $0,2 \pm 0,2$ & $0,2 \pm 0,2$ & $0,2 \pm 0,2$ \\
\hline $\begin{array}{c}\text { Linfócito } \\
(\%)\end{array}$ & $73,2 \pm 2,13$ & $65,6 \pm 1,54^{*}$ & $71,6 \pm 2,40$ & $64,8 \pm 4,42$ & $70,6 \pm 3,47$ & $72,8 \pm 1,20$ \\
\hline $\begin{array}{c}\text { Monócito } \\
(\%)\end{array}$ & $11,8 \pm 0,74$ & $11,8 \pm 0,74$ & $10,2 \pm 1,20$ & $8,6 \pm 1,43$ & $9,2 \pm 0,37$ & $11,4 \pm 1,50$ \\
\hline $\begin{array}{l}\text { Plaquetas } \\
(10 / \mathrm{mm})\end{array}$ & $\begin{array}{c}997400 \pm 6 \\
1675\end{array}$ & $\begin{array}{c}997400 \pm 616 \\
75\end{array}$ & $\begin{array}{c}487600 \pm 1985 \\
11\end{array}$ & $\begin{array}{c}810400 \pm 1159 \\
39\end{array}$ & $\begin{array}{c}974200 \pm 42 \\
925\end{array}$ & $\begin{array}{c}879200 \pm 86 \\
102\end{array}$ \\
\hline $\begin{array}{c}\text { RDW } \\
(\%)\end{array}$ & $\begin{array}{c}12,74 \pm 0,2 \\
2 \\
\end{array}$ & $12,74 \pm 0,22$ & $13,90 \pm 0,26$ & $14,56 \pm 0,11^{*}$ & $10,76 \pm 2,41$ & $12,84 \pm 0,48$ \\
\hline $\begin{array}{l}\text { VPM } \\
\left(\mathrm{mm}^{3}\right)\end{array}$ & $5,6 \pm 0,15$ & $5,6 \pm 0,15$ & $5,84 \pm 0,27$ & $5,80 \pm 0,14$ & $5,66 \pm 0,06$ & $5,62 \pm 0,07$ \\
\hline
\end{tabular}

Legenda: Os valores estão expressos em média \pm e.p.m. $(n=6)$. Teste " $t$ " de Student / Não-pareado. ${ }^{*}<0,05$. CT-I: controle do iogurte. CT-G: controle da geleia. CT-S: controle do suco. VCM: volume corpuscular médio. HCM: hemoglobina corpuscular média. CHCM: concentração de hemoglobina corpuscular média. RDW: red cell distribution (amplitude de distribuição dos glóbulos vermelhos). VPM: volume plaquetário médio.

Fonte: Pires WRCAG, et al., 2021.

\section{Avaliação anatomopatológico}

Foram avaliados o coração, estômago, fígado, pulmões e rins dos animais deste estudo. Os órgãos não apresentaram alterações macroscópicas nem microscópicas, como mudanças estruturais, inflamação aguda e crônica, esteatose, fibrose e necrose. Os cortes histológicos foram analisados pela coloração hematoxilinaeosina em microscópio óptico com aumentos de x40, x100 e x400. Nas figuras abaixo pode-se evidenciar cortes histológicos do fígado e rins mostrando que não houve alteração nesses órgãos em nenhum dos grupos estudados, apesar das alterações encontradas. 
Figura 1 - Fotomicrografias representativas de fígado dos grupos experimentais.
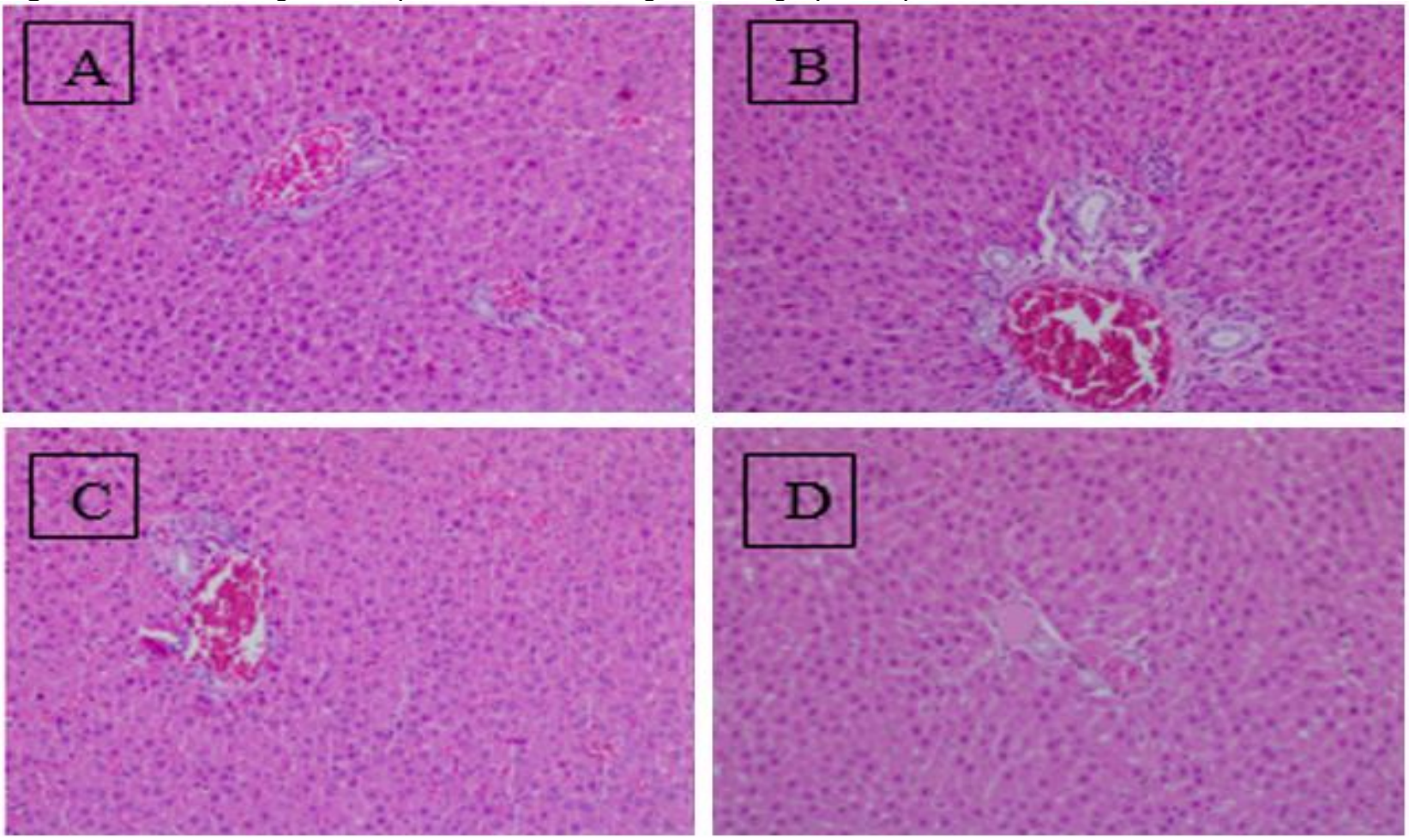

Legenda: A- Grupo controle da geléia (HE 100X); B- Grupo do suco do P. gounellei (HE 100X); C- Grupo da geléia do $P$. gounellei (HE 100X); D- Grupo do iogurte com a geléia do $P$. gounellei (HE 100X).

Fonte: Pires WRCAG, et al., 2021.

Figura 2 - Fotomicrografias representativas de rins dos grupos experimentais.
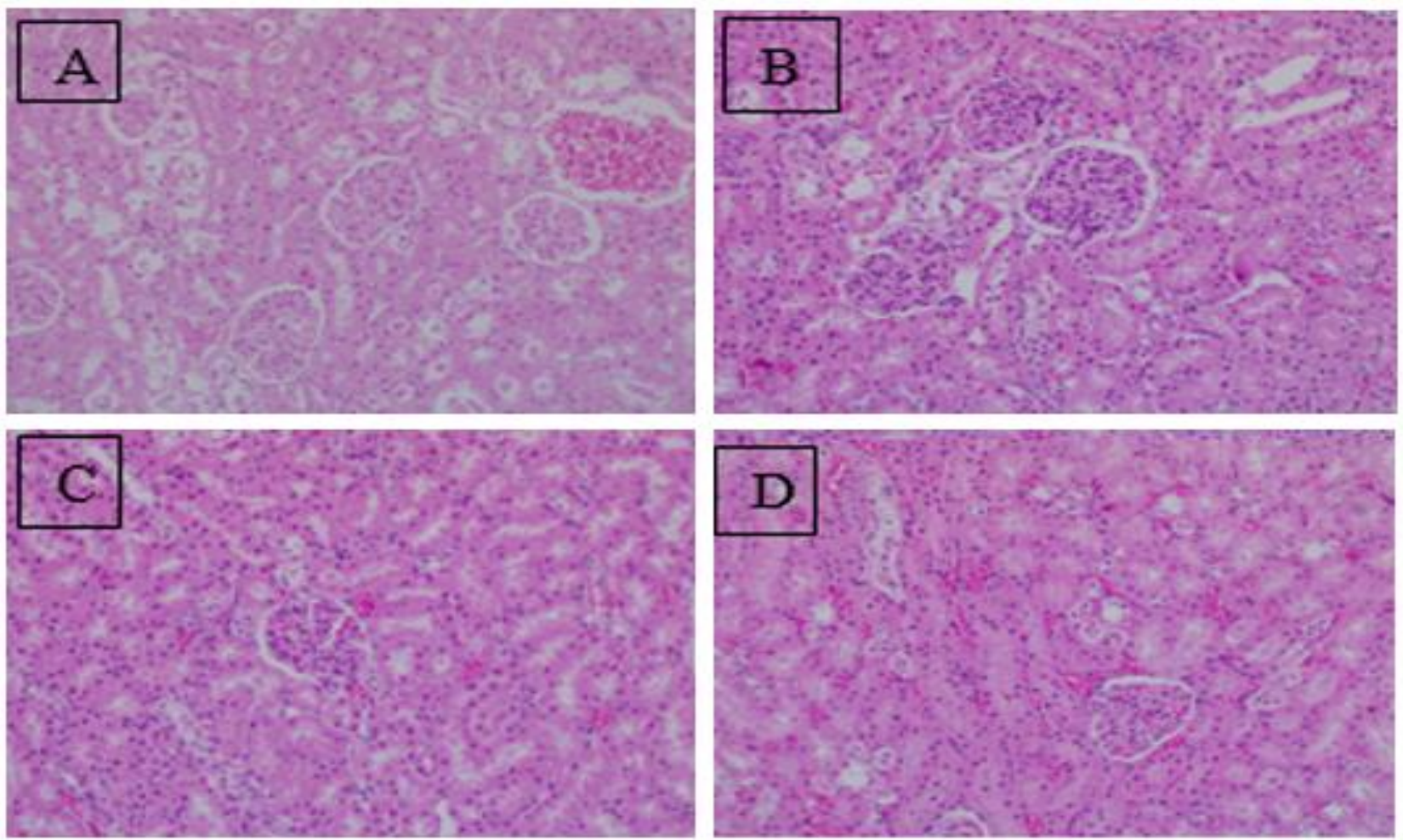

Legenda: A- Grupo controle do suco (HE 100X); B- Grupo do suco do P. gounellei (HE 100X); C- Grupo da geléia do $P$. gounellei (HE 100X); D- Grupo do iogurte com a geléia do P. gounellei (HE 100X).

Fonte: Pires WRCAG, et al., 2021. 


\section{DISCUSSÃO}

Segundo a classificação de toxicidade da Comunidade Europeia, a não ocorrência de mortes, após administração de $2000 \mathrm{mg} / \mathrm{kg}$ de um produto obtido de espécie vegetal, por via oral, indica uma provável ausência de toxicidade, porém mais dados são necessários para confirmar este achado (OLIVEIRA AM, 2019).

A ingestão de água e nutrientes são parâmetros importantes no estudo da segurança do produto, pois estes são essenciais para o funcionamento do organismo. Neste trabalho, foi evidenciada uma diminuição no consumo de água nos grupos do suco e iogurte do $P$. gounellei. Estes contêm razoável teor aquoso, sendo esta cactácea utilizada no sertão como fonte de água e alimento para animais e seres humanos. Em seu estudo, Dias GE (2015) também evidenciou diminuição no consumo de água ao utilizar o extrato etanólico do $P$. gounellei. Enquanto, Oliveira AM (2019), em seu estudo com o extrato do $P$. gounellei não evidenciou alteração no consumo da mesma.

A avaliação da massa corpórea do animal é um importante indicador para caracterização da toxicidade de uma substância. $\mathrm{O}$ ganho de peso pode ser afetado pela dificuldade de digestão dos alimentos e absorção dos nutrientes, sendo um indício de possíveis efeitos tóxicos (SANTANA LR, et al., 2014). Alguns estudos correlacionam o efeito do estresse sobre o peso corporal e ingestão de alimento, sendo verificada perda de peso mais elevada em animais expostos a mais estresse. Apesar de não haver redução no consumo de ração, os animais do grupo do iogurte apresentaram um ganho de peso menor comparado ao seu controle e aos demais grupos. Contudo, os resultados estão de acordo com o preconizado para avaliação de peso corpóreo, da OECD, (2001a), de não ultrapassar a variação de $20 \%$ do peso médio inicial.

Dentre os parâmetros bioquímicos, destaca- se o aumento da ALT, no grupo da geleia do P. gounellei. O fígado é um dos órgãos mais importantes do corpo, sendo responsável pela produção e metabolismo de várias substâncias. Lesões nos hepatócitos são detectadas através da mensuração das transaminases (ALT e AST), enzimas liberadas após rompimento destas células, e consideradas importantes marcadores para avaliação de dano hepático (ROY S, et al., 2015).

A avaliação da função hepática é feita através da mensuração de metabólitos que são produzidos ou excretados pelo órgão, como a bilirrubina e albumina. A bilirrubina é o principal componente dos pigmentos biliares e o seu aumento pode ocorrer por lesão dos hepatócitos ou bloqueio ao fluxo biliar (colestase). Já a albumina é a principal proteína circulante, sendo o fígado, o único responsável pela sua produção (GOMES BTL, et al., 2019).

$\mathrm{Na}$ hepatotoxicidade pode ocorrer lesão hepática em todas as zonas da glândula, com balonismo hepatocelular e degeneração de hepatócitos, corpos apoptóticos, pequenos focos de necrose e inflamação portal mínima (GOMES BTL, et al., 2019). Contudo, não foi observada alteração nos exames de função hepática, e nem alteração histopatológica neste órgão. O que nos leva a pensar na possibilidade de leve hepatotoxicidade pela geleia, podendo, inclusive, este efeito ser atribuído à alta dose administrada da mesma, devendo, portanto, seguir com os estudos de toxicidade subcrônica para confirmar este achado.

Estudos com extrato etanólico do $P$. gounellei não evidenciaram aumento de transaminases e nem alterações anatomopatológicas no fígado dos animais submetidos ao estudo de toxicidade aguda (OLIVEIRA AM, 2019 e DIAS GEN, 2015).

Ureia e creatinina se apresentam alteradas quando há insuficiência renal. Os rins são órgãos de extrema importância para avaliação de toxicidade, pois, quando ocorre a falência dos mesmos, a substância permanece mais tempo no organismo, devido à diminuição de sua eliminação, podendo causar danos decorrentes do aumento do seu volume sérico. A única alteração encontrada em relação à função renal foi a diminuição da ureia no grupo do suco do $P$. gounellei, o que poderia caracterizar uma possível proteção renal. Dias GEN (2015) em seu estudo com o extrato do $P$. gounellei identificou um discreto aumento da ureia e diminuição da creatinina. Enquanto que Oliveira LJ (2011) não identificou modificações nestes parâmetros.

A análise dos parâmetros hematológicos é de relevante importância para avaliação dos riscos de determinadas substâncias quando administrada em seres humanos, pois o sistema hematológico é um alvo bastante susceptível ao ataque de substâncias tóxicas (LIMA FEG et al., 2018). 
Hemácias são unidades morfológicas da série vermelha sanguínea, cuja função é transportar o oxigênio aos tecidos e recolher o gás carbônico. VCM, HCM e RDW são índices hematimétricos utilizados para avaliar estas células. RDW mensura a variação de tamanho entre as hemácias, dessa forma, quando está elevado, significa que as mesmas possuem diferentes tamanhos. O aumento do VCM indica que as hemácias estão maiores que o habitual, pois ele avalia o tamanho médio dos glóbulos vermelhos (DIAS GEN, 2015).

No grupo da geleia do $P$. gounellei ocorreu um aumento estatisticamente significativo no RDW e VCM. Contudo, estas alterações ainda se encontram próximas aos valores de referência e não houve modificação dos demais parâmetros indicativos de anemia (hemácias, hemoglobina e hematócrito). Conclui-se, assim, que os glóbulos vermelhos apresentam alteração em seu tamanho, com predomínio de macrócitos (hemácias maiores que as habituais), porém, em número ainda normais. Geralmente, em humanos, os macrócitos são vistos em deficiência de vitaminas como ácido fólico e vitamina B12, sugerindo-se, assim, avaliações desses parâmetros para melhor investigação deste achado (LIMA FEG et al., 2018). Como exemplo, tem-se o metotrexato, medicamento utilizado em doenças autoimunes e neoplasias, que tem como efeito inibir o folato, causando entre outras alterações macrocitose e anemia macrocítica.

Um estudo feito por Oliveira LJ (2011), avaliou os parâmetros hematológicos em um bioensaio com $P$. gounellei e não encontrou nenhuma alteração. No grupo do iogurte do $P$. gounellei foi observado aumento do RDW e diminuição do hematócrito. O hematócrito avalia a porcentagem de volume ocupada pelas hemácias no total de sangue. Ele diminui na anemia, devido à redução na produção ou aumento da destruição de hemácias, e quando há hemorragia. Porém, esta alteração não foi acompanhada por queda de hemoglobina e nem diminuição do número de hemácias, o que a princípio seria contraditório (LIMA FEG et al., 2018). Constata-se, que, estudos adicionais são necessários para confirmar estes achados.

A linfopenia caracteriza-se pela diminuição dos linfócitos, um dos subtipos de leucócitos, células responsáveis pela defesa do nosso organismo. Essa alteração foi observada no grupo que recebeu o iogurte da geleia do $P$. gounellei, podendo ser relacionada a diversas causas, dentre elas o estresse ou a alta dose administrada da substância. Estudos prévios não mostraram alterações dos glóbulos brancos na avaliação dos parâmetros hematológicos (OLIVEIRA LJ, 2011 e DIAS GE, 2015).

Estas variações apesar de serem estatisticamente significativas não possuem relevância clínica, pois os valores estão próximos aos de referência, podendo tais diferenças serem explicadas pela variabilidade biológica entre os ratos (CASTELLO BRANCO AC, et al., 2011).

O cálcio e fósforo estão presentes no esqueleto humano, e têm em comum, o metabolismo associado à vitamina $\mathrm{D}$ e paratormônio. Por meio destes e com auxílio do rim, intestino e esqueleto, processa-se a homeostase destes íons (SILVA ICG, et al., 2021).

O magnésio é o segundo cátion intracelular mais abundante, tendo participação na maioria dos processos metabólicos (BEZERRIL FF, et al., 2021). Sua concentração sérica é resultado de um balanço na ingestão/absorção, biodistribuição e excreção. Hipermagnesemia é encontrada em casos de insuficiência renal e no uso de laxativos contendo magnésio. Já a hipomagnesemia tem em suas causas doenças do trato gastrintestinal, como síndromes disabsortivas e pós-ressecções de intestino delgado, podendo também ser induzida por alimentação enteral prolongada sem suplementação de magnésio e pelo uso excessivo de laxativos.

No grupo do suco do $P$. gounellei, houve um aumento dos níveis de magnésio e no grupo do iogurte do $P$. gounellei ocorreu uma diminuição destes níveis em relação ao controle. Importante avaliar que apesar destas alterações, os valores do magnésio permaneceram ainda dentro da normalidade. Isto pode ter ocorrido pelas propriedades do iogurte em modificar a microbiota intestinal, alterando o processo de digestão e absorção de nutrientes (CARVALHO POAA, et al., 2021). Neste último grupo, além da hipomagnesemia ocorreu também uma hipofosfatemia, o que ajuda a corroborar nossa hipótese. Existem alguns mecanismos para a ocorrência da hipofosfatemia, que são: redistribuição do fósforo do meio extracelular para o meio intracelular, diminuição da absorção intestinal e aumento da excreção urinária.

Importante observar que os três minerais, acima mencionados, dependem da absorção intestinal para manter os seus valores de normalidades. Como foi visto anteriormente, estudos com o cladódio do P.gounellei 
mostraram a presença de fibras consideradas prebióticas, que servem como alimento para as bactérias intestinais, resultando em possíveis alterações na absorção destes minerais (BEZERRIL FF, et al., 2017; RIBEIRO TS, et al., 2020).

O cloro é essencial no equilíbrio hídrico e do sódio, e na regulação da pressão osmótica. A hipercloremia é caracterizada por um excesso de cloreto, causada por desidratação grave, falência renal e hemodiálise. No grupo do suco do P.gounellei foi evidenciado aumento do cloro em relação ao seu controle, porém, os valores ainda permaneceram dentro da normalidade para esta espécie (SILVA ICG, et al., 2021).

Apesar de nenhum órgão ter apresentado alterações macroscópicas após a autopsia dos animais, todos foram enviados para exame histopatológico. Nenhum deles apresentou alterações em sua microscopia, portanto não foi evidenciado nenhum tipo de inflamação, seja ela aguda ou crônica, fibrose, esteatose ou necrose.

\section{CONCLUSÃO}

Conclui-se, assim, que o suco e o iogurte da geleia do $P$. gounellei possuem baixa toxicidade. Em relação à geleia do $P$. gounellei, pode-se inferir que mais estudos são necessários para descartar hepatotoxicidade, já que a mesma ocasionou elevação discreta de ALT, o que pode ter sido relacionada à alta dose administrada. Como limitação ao estudo ressalta-se a curta duração do mesmo, devendo-se levar em consideração, que para afirmar a segurança do uso das substâncias em estudo, faz-se necessário a realização de ensaios crônicos, com o intuito de avaliar o seu efeito a longo prazo e melhor caracterizar possíveis efeitos tóxicos. A importância desse projeto de pesquisa advém da necessidade de se encontrar novos agentes nutritivos, farmacologicamente ativos e seguros, etapa fundamental na cadeia de produção de medicamento, e relevante para prosseguir com os estudos clínicos em humanos, contribuindo assim com sua utilização como forma de prevenção e cuidado com a saúde.

\section{AGRADECIMENTOS}

Agradecemos a Fabrícia de França Bezerril por contribuir com este projeto auxiliando na produção do suco, iogurte e geléia do Pilosocereus gounellei.

\section{REFERÊNCIAS}

1- BEZERRIL FF. Caracterização nutricional e de compostos bioativos do Xique-Xique (Pilosocereus gounellei (A. Weber ex. K. Schum.) Bly. ex Rowl.). Dissertação (Mestrado em Ciência e Tecnologia de Alimento). Departamento de Nutrição. Universidade Federal da Paraíba, João Pessoa, 2017; 77 p.

2- BEZERRIL FF, et al. Pilosocereus gounellei (xique xique) jam is source of fibers and mineral and improves the nutrionitional value and the technological properties of goat milk yogurt. LWT- Food Science and Technology, 2021; 139.

3- BRASIL. Agência Nacional de Vigilância Sanitária - ANVISA. Guia para condução de estudos não clínicos e toxicologia e segurança farmacológica necessários ao desenvolvimento de medicamentos. Brasília-DF 2013. Disponível em: www.anvisa.gov.br. Acessado em 01 de junho de 2021.

4- BRAVO FILHO ES. Diversidade, Etnobotânica e Propagação de cabeça-de-frade (Melocactus Link \& Otto CACTACEAE) no Estado de Sergipe. Dissertação (Mestrado em Desenvolvimento e Meio Ambiente) Universidade Federal de Sergipe, São Cristovão, 2014; 161 p.

5- CARVALHO POAA, et al. Nutritional potential and bioactive compounds of xique-xique juice: An unconventional food plant from Semiarid Brazilian. J Food Process Preserv, 2021; 45: e15265.

6- CASTELLO BRANCO ACS, et al. Parâmetros bioquímicos e hematológicos de ratos Wistar e camundongos Swiss do Biotério Professor Thomas George. Revista Brasileira de Ciências da Saúde, 2011; 15 (2), 209-14.

7- CAVALCANTI NB, RESENDE GM. Efeito de diferentes substratos no desenvolvimento de mandacaru (Cereus jamacaru P. DC.), facheiro (Pilosocereus pachycladus RITTER), xiquexique (Pilosocereus gounellei (A. WEBWR EX K. SCHUM.) BLY. EX ROWL.) e coroa-de-frade (Melocactus bahiensis BRITTON). Caatinga, 2007; 20 (1): $28-35$.

8- CHAVES EMF, BARROS RFM. Cactáceas: Recurso alimentar emergencial no semiárido, Nordeste do Brasil. Gaia Scientia, 2015; 9, 129-135.

9- DIAS GEN. Avaliação da toxicidade não clínica do extrato e etanólico bruto de Pilosocereus gounellei (F.A.C Weber) em ratos. Dissertação (Doutorado em Farmacologia). Universidade Federal da Paraíba, João Pessoa, 2015; 79p. 
10-GOLDIM JR. A Avaliação Ética Da Investigação Científica de Novas Drogas: A Importância da Caracterização Adequada das Fases de Pesquisa. Revista HCPA, 2007; 27; 66-73.

11-GOMES BTL, et al. Avaliação da função hepática:uma revisão bibliográfica. Bio Medicina, $2019 ; 4$ (1).

12- LIMA, FEG. Perfil bioquímico e hematológico de ratos e camundongos do biotério central da Universidade Federal do Ceará. Dissertação (Mestrado Profissional em Farmacologia Clínica) - Faculdade de Medicina, Universidade Federal do Ceará, Fortaleza, 2018; 75p.

13- LI X, et al. Acute and subacute toxicity of ethanol extracts from Salvia przewalskii Maxim in rodents. Journal of Ethnopharmacology, 2010; 131: 110-115.

14-LUCENA CM, et al. Conhecimento local sobre cactáceas em comunidades rurais na mesorregião do sertão da Paraíba (Nordeste, Brasil). Universidade Federal da Paraíba. 2012; 25(3): 282-288.

15-MACIEL KS, et al. New Alcamide and anti- oxidant activity of Pilosocereus gounellei A. Weber ex K. Schum. Bly. ex Rowl. (Cactaceae). Molecules, 2016; 21: 1-13.

16-MENEZES MOT, RIBEIRO-SILVA S. Cactáceas do Ceará, Brasil: prioridades para a conservação. Revista Gaia Scie, 2015; 9(2): 67-76.

17-OECD. Organização para Cooperação Econômica e Desenvolvimento (Oragnization for Economic Cooperation and Development). Guideline 423: Acute Oral Toxicity Method. Guideline for testing of Chemicals,2001a. Disponível: www.oecd-ilibrary.org. Acessado em: 01 de junho de 2021.

18-OECD. Organização para Cooperação Econômica e Desenvolvimento (Oragnization for Economic Cooperation and Development). Guideline 420: Acute Oral Toxicity -Fixed Dose Procedure Method. Guideline for testing of Chemicals, 2001b. Disponível: www.oecd-ilibrary.org. Acessado em: 01 de junho de 2021.

19-OLIVEIRA LJ. Bioensaios de Pilosoceresu gounellei (Weber) Byles e Rowley (Parâmetros fitoquímicos, toxicológicos, microbiológicos, hematológicos e bioquímicos). Dissertação (Mestrado em Ciências Farmacêuticas). Universidade Federal de Pernambuco, Recife, 2011; 88p.

20-OLIVEIRA AM. Avaliação da toxicidade aguda e subaguda e efeitos nociceptivos, anti pirético e anti obesidade de extrato de caule de P. gounellei. Tese (Doutorado em Bioquímica e Fisiologia). Universidade Federal de Pernambuco, Recife, 2019.

21-PINTO NCC, SCIO E. The biological activities and chemical composition of Pereskia species (Cactaceae) - a review. Plant Foods for Human Nutrition, 2014; 69(3): 189-195.

22-RIBEIRO TS, et al. In vitro evaluation of potential prebiotic effects of a freeze-dried juice from Pilosocereus gounellei (A. Weber ex K. Schum. Bly. Ex Rowl) cladodes, an unconventional edible plant from Caatinga biome. 3 Biotech, 2020; 10: $1-9$.

23-ROCHA FILHO CAF. Investigação do caule do xique-xique( $P$.gounellel) como fonte de inibidor de tripsina com ação antibacteriana. Dissertação (Mestrado emCiências Biológicas). Universidade Federal de Pernambuco, Recife, 2015; $58 \mathrm{p}$.

24-ROY S, et al. Synthesis, Characterization, Antioxidant Status, and Toxicity Study of Vanadium-Rutin Complex in Balb/c Mice. Biol Trace Elem Res. 2015; 66: 83-200.

25-SANTANA LCLR, et al. Estudo das propriedades físico-químicas e avaliação da toxicidade aguda do extrato etanólico das folhas de Mikania glomerata Sprengel. Revista Brasileira de Plantas Medicinais, 2014; 16(3): 670-678.

26-SANTOS AG, PEREIRA LC. Utilização de métodos alternativos à experimentação animal em pesquisa. Revista da Sociedade Brasileira de Ciência em Animais de Laboratório, 2016; 4(1): $22-31$.

27-SILVA VA. Diversidade de uso das cactáceas no nordeste do Brasil: uma revisão. Revista Gaia Scie, 2015; 9(2): 137154.

28-SOUSA GA, et al. Gastroprotective effect of ethanol extracts of cladodes and roots of Pilosocereus gounellei (A. Weber ex K. Schum.) Bly. Ex Rowl (Cactaceae) on experimental ulcer models. Journal of Ethnopharmacology, 2018; 218: 100-108.

29-VERRI AM, et al. Testes citogenéticos na avaliação da genotoxicidade de produtos naturais provindos de plantas medicinais. Revista Uningá Review, 2017; 30(1): 55-6.

30-SILVA ICG, et al. Deficiency of vitamins and minerals: the role of technology in health prevention: an integrative review. Research, Society and Development, 2021; 9(10): e4129108700. 